\title{
FANNING OUT OF THE SOLAR $f$-MODE IN THE PRESENCE OF NON-UNIFORM MAGNETIC FIELDS?
}

\author{
Nishant K. Singh ${ }^{1}$, Axel Brandenburg $^{1,2}$, AND Matthias RheinhardT ${ }^{3}$ \\ ${ }^{1}$ Nordita, KTH Royal Institute of Technology and Stockholm University, Roslagstullsbacken 23, SE-10691 Stockholm, Sweden; nishant@nordita.org \\ ${ }^{2}$ Department of Astronomy, Stockholm University, SE-10691 Stockholm, Sweden \\ ${ }^{3}$ Physics Department, Gustaf Hällströmin katu 2a, PO Box 64, FI-00014 University of Helsinki, Helsinki, Finland \\ Received 2014 July 1; accepted 2014 September 10; published 2014 October 16
}

\begin{abstract}
We show that in the presence of a magnetic field that is varying harmonically in space, the fundamental mode, or $f$-mode, in a stratified layer is altered in such a way that it fans out in the diagnostic $k \omega$ diagram, with mode power also within the fan. In our simulations, the surface is defined by a temperature and density jump in a piecewise isothermal layer. Unlike our previous work (Singh et al. 2014), where a uniform magnetic field was considered, here we employ a non-uniform magnetic field together with hydromagnetic turbulence at length scales much smaller than those of the magnetic field. The expansion of the $f$-mode is stronger for fields confined to the layer below the surface. In some of those cases, the $k \omega$ diagram also reveals a new class of low-frequency vertical stripes at multiples of twice the horizontal wavenumber of the background magnetic field. We argue that the study of the $f$-mode expansion might be a new and sensitive tool to determine subsurface magnetic fields with azimuthal or other horizontal periodicity.
\end{abstract}

Key words: magnetohydrodynamics (MHD) - Sun: helioseismology - turbulence - waves

Online-only material: color figures

\section{INTRODUCTION}

For several decades, helioseismology has provided information about the solar interior through detailed investigations of sound or pressure waves, generally referred to as $p$-modes. While internal gravity waves, or $g$-modes, are evanescent in the convection zone and hence not seen in the Sun, the so-called surface or fundamental mode ( $f$-mode) is observable. This mode is just like deep water waves. In that case, it is well known that the presence of surface tension leads to additional modes known as capillary waves (Dias \& Kharif 1999). Such modes do not exist on gaseous interfaces, but magnetic fields could mimic the effects of surface tension and thus lead to characteristic alterations of the $f$-mode, which could potentially be used to determine properties of the underlying magnetic field. Earlier work has indeed shown that both vertical and horizontal uniform magnetic fields have a strong effect on the $f$-mode (Singh et al. 2014, hereafter SBCR), but, obviously, the assumption of a uniform magnetic field is unrealistic.

The goal of local helioseismology using the $f$-mode (Hanasoge et al. 2008; Daiffallah et al. 2011; Felipe et al. 2012,2013 ) is to determine the local structure of the underlying magnetic field. Such techniques might be more sensitive than local techniques employing only $p$-modes (see, e.g., Gizon et al. 2010). Our aim is to determine the structure of sunspot magnetic fields and to decide whether they have emerged as isolated flux tubes from deeper layers (Parker 1975; Caligari et al. 1995), as expected from the flux transport dynamo paradigm. An alternative approach to solar magnetism presumes that the dynamo is a distributed one operating throughout the entire convection zone and not just at its bottom, and that sunspots are merely localized flux concentrations near the surface. This approach was discussed in some detail by Brandenburg (2005), who mentioned the negative effective magnetic pressure instability (Kleeorin et al. 1996) and the local suppression of turbulent heat transport (Kitchatinov \& Mazur 2000) as possible agents facilitating the formation of such magnetic flux concentrations. He also discussed magnetic flux segregation into magnetized and unmagnetized regions (Tao et al. 1998) as a mechanism involved in the formation of active regions. In those two instabilities, the magnetic field experiences a local concentration near the surface. In the horizontal plane, the field shows then a periodic pattern that also plays a role in the motivation of the field pattern chosen for the present investigation.

\section{MODEL SETUP AND MOTIVATION}

Instead of solving an eigenvalue problem to obtain the mode frequencies, we infer them from direct numerical simulations via diagnostic wavenumber-frequency diagrams, as observers would do. Our model is close to that studied in SBCR with a piecewise isothermal setup consisting of a lower cooler layer ("bulk" with thickness $L_{z \mathrm{~d}}$ ) and a hotter upper one ("corona" with thickness $L_{z u}$ ), referred to by subscripts "d" and "u," respectively. Their interface is placed at $z=0$. The only difference is that we include here an electromotive force to maintain the now non-uniform background field. We solve the basic hydromagnetic equations,

$$
\begin{gathered}
\frac{D \ln \rho}{D t}=-\nabla \cdot \boldsymbol{u}, \\
\frac{D \boldsymbol{u}}{D t}=\boldsymbol{f}+\boldsymbol{g}+\frac{1}{\rho}(\boldsymbol{J} \times \boldsymbol{B}-\nabla p+\nabla \cdot 2 v \rho \mathbf{S}), \\
T \frac{D s}{D t}=2 \nu \mathbf{S}^{2}+\frac{\mu_{0} \eta}{\rho} \boldsymbol{J}^{2}-(\gamma-1) c_{p} \frac{T-T_{\mathrm{d}, \mathrm{u}}}{\tau_{\mathrm{c}}}, \\
\frac{\partial \boldsymbol{A}}{\partial t}=\boldsymbol{u} \times \boldsymbol{B}+\mathcal{E}_{0}-\eta \mu_{0} \boldsymbol{J},
\end{gathered}
$$

where $\boldsymbol{u}$ is the velocity, $D / D t=\partial / \partial t+\boldsymbol{u} \cdot \boldsymbol{\nabla}$ is the advective time derivative, $\boldsymbol{f}$ is a forcing function specified below, $\boldsymbol{g}=$ $(0,0,-g)$ is the gravitational acceleration, $S_{i j}=(1 / 2)\left(u_{i, j}+\right.$ $\left.u_{j, i}\right)-(1 / 3) \delta_{i j} \boldsymbol{\nabla} \cdot \boldsymbol{u}$ is the traceless rate of strain tensor, 


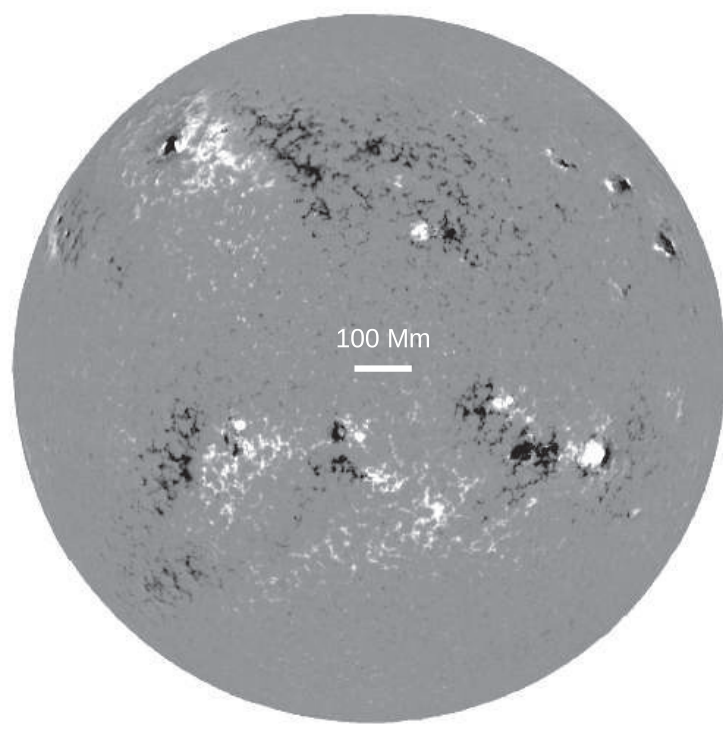

Figure 1. Full disk solar magnetogram showing the line of sight magnetic field during a very active phase. Light (dark) shades correspond to positive (negative) values. Note the characteristic wavelength $\approx 100 \mathrm{Mm}$ of regular sign changes.

where commas denote partial differentiation, $v=$ const is the kinematic viscosity, $s$ is the specific entropy, $\boldsymbol{A}$ is the magnetic vector potential, $\boldsymbol{B}=\boldsymbol{\nabla} \times \boldsymbol{A}$ is the magnetic field, $\boldsymbol{J}=\mu_{0}^{-1} \nabla \times \boldsymbol{B}$ is the current density, $\mathcal{E}_{0}$ is an external electromotive force specified below, $\eta=$ const is the magnetic diffusivity, $\mu_{0}$ is the vacuum permeability, $\gamma=c_{\mathrm{p}} / c_{\mathrm{v}}$ is the ratio of specific heats at constant pressure and density, respectively, and $T$ is the temperature. The last term in Equation (3), being of relaxation type, is to guarantee that the temperature is, on average, constant in either subdomain and equal to $T_{\mathrm{d}}$ and $T_{\mathrm{u}}$, respectively. For the relaxation rate $\tau_{\mathrm{c}}^{-1}$, we choose, as in SBCR, $g /\left(2 c_{\mathrm{sd}}\right)$ in $z>0$ and zero in $z<0$. (Dropping the relaxation term in the bulk is preferable as it allows the flow to evolve more freely while causing only a slow drift of $T$ away from $T_{\mathrm{d}}$. In the corona, on the other hand, our choice of $\tau_{\mathrm{c}}$ is sufficient to keep deviations of $T$ from $T_{\mathrm{u}}$ small without affecting the oscillations markedly.)

The adiabatic sound speeds in the upper and lower layers are referred to as $c_{\mathrm{su}}$ and $c_{\mathrm{sd}}$, respectively. In most of the cases, we assume a temperature jump $q=T_{\mathrm{d}} / T_{\mathrm{u}}=c_{\mathrm{sd}}^{2} / c_{\mathrm{su}}^{2}=$ $\rho_{\mathrm{u}}(0) / \rho_{\mathrm{d}}(0)$ of about one-tenth, which means that at the interface the density changes by the same factor, thus allowing for the $f$-mode to appear. A random flow is driven in the lower layer $(z<0)$ by applying a solenoidal nonhelical forcing with a wavenumber that is much larger than the lowest one fitting into the domain.

This is a minimalistic model as it employs just those ingredients that are necessary to produce the desired magnetically affected $f$-mode: the interface, maintained by thermal relaxation, gravity, and the electromotive force owing to the non-uniformity of the background field. Viscosity and magnetic diffusivity are merely needed for numerical stability, but then a random forcing is necessary for mode excitation.

We normalize the length scales by $L_{0}=\gamma H_{\mathrm{d}}=c_{\mathrm{sd}}^{2} / g$, where $H_{\mathrm{d}}$ is the pressure scale height in the bulk, while frequencies are normalized by $\omega_{0}=g / c_{\mathrm{sd}}$. Quantities normalized this way are indicated by tildae.

In a customary $k_{x}-\omega$ diagram (referred to simply as $k \omega$ diagram), we show the amplitude of the Fourier transform of the vertical velocity $u_{z}$, taken from the interface at $z=0$, as a function of $k_{x}$ and $\omega$. As the Fourier transform $\hat{u}_{z}\left(k_{x}, \omega\right)$ has the dimension of length squared, we construct the dimensionless quantity

$$
\widetilde{P}\left(\omega, k_{x}\right)=\frac{\left|\hat{u}_{z}\right|}{\mathcal{D}^{2}}=\frac{\left|\hat{u}_{z}\right|}{L_{0}^{2}} \frac{c_{\mathrm{sd}}^{2}}{u_{\mathrm{rms}, \mathrm{d}}^{2}},
$$

where $u_{\mathrm{rms}, \mathrm{d}}$ represents the rms value of the random motions in the bulk and $\mathcal{D}=u_{\mathrm{rms}, \mathrm{d}} / \omega_{0}$ is the distance traveled with speed $u_{\text {rms,d }}$ in a time $\omega_{0}^{-1}$. The fluid Reynolds and Mach numbers of the flow are defined as $\operatorname{Re}=u_{\mathrm{rms}, \mathrm{d}} /\left(\nu k_{\mathrm{f}}\right)$ and $\mathrm{Ma}=u_{\mathrm{rms}, \mathrm{d}} / c_{\mathrm{sd}}$, respectively.

As in SBCR, we employ a two-dimensional model in $x$ and $z$, ignoring any variations in the $y$ direction. The domain is of size $L_{x} \times L_{z}=8 \pi L_{0} \times \pi L_{0}$, where $L_{x}$ and $L_{z}$ denote its horizontal and vertical extents, respectively. For the boundary conditions, we adopt a perfect conductor and vanishing stress at the top and bottom of the domain and periodicity in the $x$-direction. All calculations are performed with the PENCIL CODE. ${ }^{4}$

As discussed at the end of Section 1, we produce a steady magnetic field $\boldsymbol{B}_{0}$ by applying a constant external electromotive force $\mathcal{E}_{0}$ with a harmonic spatial variation

$$
\mathcal{E}_{0}=\hat{\mathcal{E}}_{0} \cos \left(k_{x}^{B} x\right) \cos \left(k_{z}^{B} z\right) \boldsymbol{e}_{y} .
$$

Our choice of a sinusoidally varying magnetic field can be motivated by looking at a solar magnetogram showing a regular pattern of alternating positive and negative vertical field along the azimuthal direction (Figure 1). However, the stationary magnetic field that emerges in the domain is the result of the combined effects of $\mathcal{E}_{0}$ and the Lorentz force of $\boldsymbol{B}_{0}$. Given that initially, when the fluid is still at rest, $\mathcal{E}_{0}$ generates a field resembling that of an array of (thick) straight wires in the $y$-direction, the Lorentz force will tend to compress these field vortices (rolls) and hence accumulate fluid within them. Consequently, they have to sink to a position where their excess weight is compensated by the magnetic pressure gradient of the field concentrated between the rolls and the lower boundary. Finally, the overall state adjusts to a steady MHD equilibrium $\left(\boldsymbol{U}_{0}, \rho_{0}, \boldsymbol{B}_{0}\right)$, which can qualitatively be characterized by the spectra of its fields with respect to $x$. Assuming $k_{x}^{B}=N k_{1}$, where $k_{1}=2 \pi / L_{x}$ (in this work, $k_{1} L_{0}=0.25$ ), the spectra of $\boldsymbol{U}_{0}$ and $\rho_{0}$ are given by $\pm 2 m N k_{1}$ and that of $\boldsymbol{B}_{0}$ by $\pm(2 m+1) N k_{1}$ with $m=0,1,2, \ldots$ In Figure 2, we show visualizations of the background field $\boldsymbol{B}_{0}$ for all runs discussed in the results section; see Table 1.

We define the $z$ dependent rms Alfvén speed, $v_{\mathrm{A}}(z)$, and the quantity $\beta$, characterizing the subsurface concentration of $v_{\mathrm{A}}(z)$ as

$$
v_{\mathrm{A}}(z)=\sqrt{\frac{\left\langle B^{2}\right\rangle_{x}(z)}{\mu_{0}\langle\rho\rangle_{x}(z)}}, \quad \beta=\left(\max _{z \leqslant 0} v_{\mathrm{A}}(z)\right) / c_{\mathrm{sd}} .
$$

In Figure 3, we show the variation of $v_{\mathrm{A}}(z) / c_{\mathrm{s}}(z)$ with $z$ for all runs; more details are given in Table 1 , where $z_{\mathrm{m}}$ is the position of the maximum in Equation (7). We also show that the $f$-mode asymmetry, characterized by the quantity $\mathcal{A}_{\mathrm{f}}$ (defined below), increases with $z_{\mathrm{m}}$ and varies only weakly with $\beta$.

For a uniform magnetic field imposed in the $x$-direction, the $f$-mode frequency is well described by the dispersion relation (Chandrasekhar 1961; Miles \& Roberts 1992; Miles et al. 1992)

$$
\omega_{\mathrm{fm}}^{2}=c_{\mathrm{fm}}^{2} k_{x}^{2}+g k_{x} \frac{1-q}{1+q},
$$

\footnotetext{
4 http://pencil-code.googlecode.com/
} 

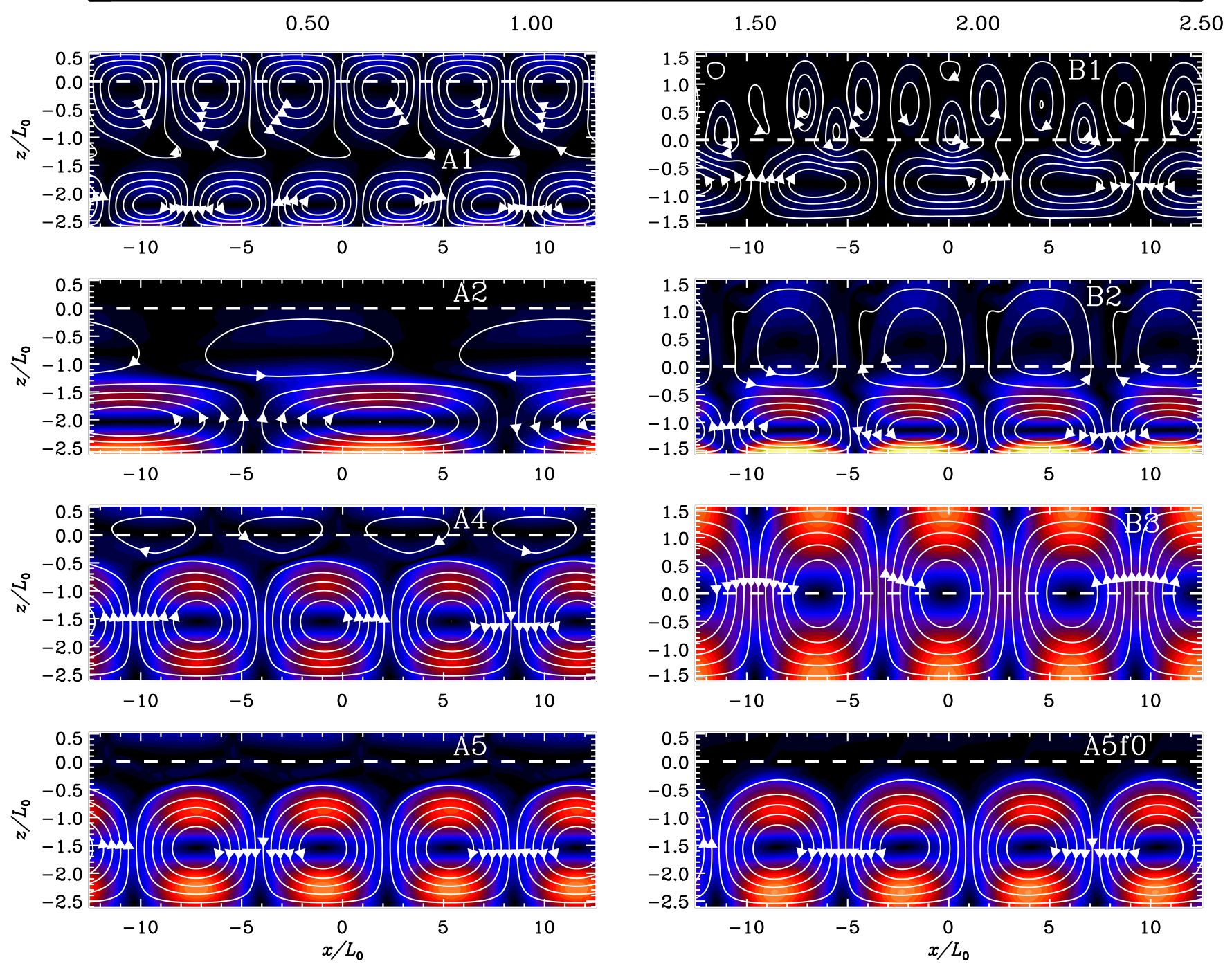

Figure 2. Flux lines of the saturated magnetic background fields of the runs in Table 1 . Colors: modulus of $\boldsymbol{B}$. $|\boldsymbol{B}|$ in units of $0.65 c_{\mathrm{sd}} \sqrt{\mu_{0} \rho_{\mathrm{d}}(0)}$.

(A color version of this figure is available in the online journal.)

Table 1

Summary of Simulations

\begin{tabular}{lcccccccc}
\hline \hline Run & $\widetilde{k}_{x}^{B}$ & $\widetilde{k}_{z}^{B}$ & $\beta$ & $\widetilde{z}_{\mathrm{m}}$ & $\Delta \omega_{\mathrm{f}+} / \omega_{\mathrm{f}}$ & $\Delta \omega_{\mathrm{f}-} / \omega_{\mathrm{f}}$ & $\operatorname{Re}$ & $\mathrm{Ma}$ \\
\hline A1 & 0.75 & 2.5 & 0.074 & 0 & $\ldots$ & $\ldots$ & 4.95 & 0.0198 \\
$\mathrm{~A} 2$ & 0.25 & 3.0 & 0.118 & -1.5 & 0.33 & 0.23 & 9.67 & 0.0201 \\
$\mathrm{~A} 4$ & 0.50 & 2.0 & 0.234 & -0.8 & 0.33 & 0.37 & 0.79 & 0.0032 \\
$\mathrm{~A} 5$ & 0.50 & 2.0 & 0.285 & -0.8 & 0.44 & 0.41 & 1.15 & 0.0046 \\
$\mathrm{~A} 5 \mathrm{f0} 0^{\mathrm{a}}$ & 0.50 & 2.0 & 0.278 & -0.75 & 0.11 & 0.0 & 0.74 & 0.0030 \\
$\mathrm{~B} 1$ & 0.50 & 4.0 & 0.034 & -0.3 & $\ldots$ & $\ldots$ & 0.05 & 0.0010 \\
$\mathrm{~B} 2$ & 0.50 & 2.0 & 0.104 & -0.7 & 0.33 & 0.30 & 1.06 & 0.0042 \\
B3 & 0.50 & 1.0 & 0.127 & 0 & $\ldots$ & $\ldots$ & 0.03 & 0.0006 \\
\hline
\end{tabular}

Notes.

All runs have $q=0.1, v / \eta=1$, and $\widetilde{k}_{\mathrm{f}}=20 . \Delta \omega_{\mathrm{f} \pm} / \omega_{\mathrm{f}}$ are evaluated at $\widetilde{k}_{x}=9$.

a No random forcing.

where $c_{\mathrm{fm}}^{2}=2 \rho_{\mathrm{d}}(0) v_{\mathrm{A} x \mathrm{~d}}^{2} /\left(\rho_{\mathrm{d}}(0)+\rho_{\mathrm{u}}(0)\right)$ with $v_{\mathrm{A} x \mathrm{~d}}$ being the Alfvén speed just below the interface; see Equation (21) of SBCR. Here, the second term on the right-hand side represents the (square of the) classical, unmagnetized $f$-mode frequency, to which the first term, being the magnetic contribution, always adds. Thus, horizontal magnetic fields lead to an increase in the $f$-mode frequency, but, as discussed by Murawski \& Roberts (1993), turbulence without magnetic field leads to a decrease. SBCR found that strong vertical magnetic fields also lead to a decrease of the $f$-mode frequency for sufficiently large $\widetilde{k}_{x}$.

Berton \& Heyvaerts (1987) analyzed the alterations of the $p$-mode frequencies in the presence of a non-uniform (piecewise uniform periodic) magnetic field, but they did not consider $f$-modes. It would be important to determine how a harmonic magnetic field affects the $f$-mode frequencies, but such calculations have not yet been done. Some qualitative insight can be gained from an analysis of the possible eigensolution spectra. The coefficients in the linearized MHD equations for the perturbations $\boldsymbol{u}^{\prime}, \rho^{\prime}$, and $\boldsymbol{B}^{\prime}$, which are essentially determined by the background fields $\boldsymbol{U}_{0}, \rho_{0}$, and $\boldsymbol{B}_{0}$, are periodic in $x$ (or constant). Hence, the eigenmodes must, in general, comprise an infinitude of $x$ wavenumbers. With the spectra of the background fields derived above, we expect for $\boldsymbol{u}^{\prime}$ and $\rho^{\prime}$ nonvanishing spectral amplitudes at $( \pm 2 m N \pm l) k_{1}$, but for $\boldsymbol{B}^{\prime}$ at $( \pm(2 m+$ 1) $N \pm l) k_{1}$, where $m=0,1,2, \ldots$, and $l$ is a fixed integer, $0 \leqslant l \leqslant N / 2$. 

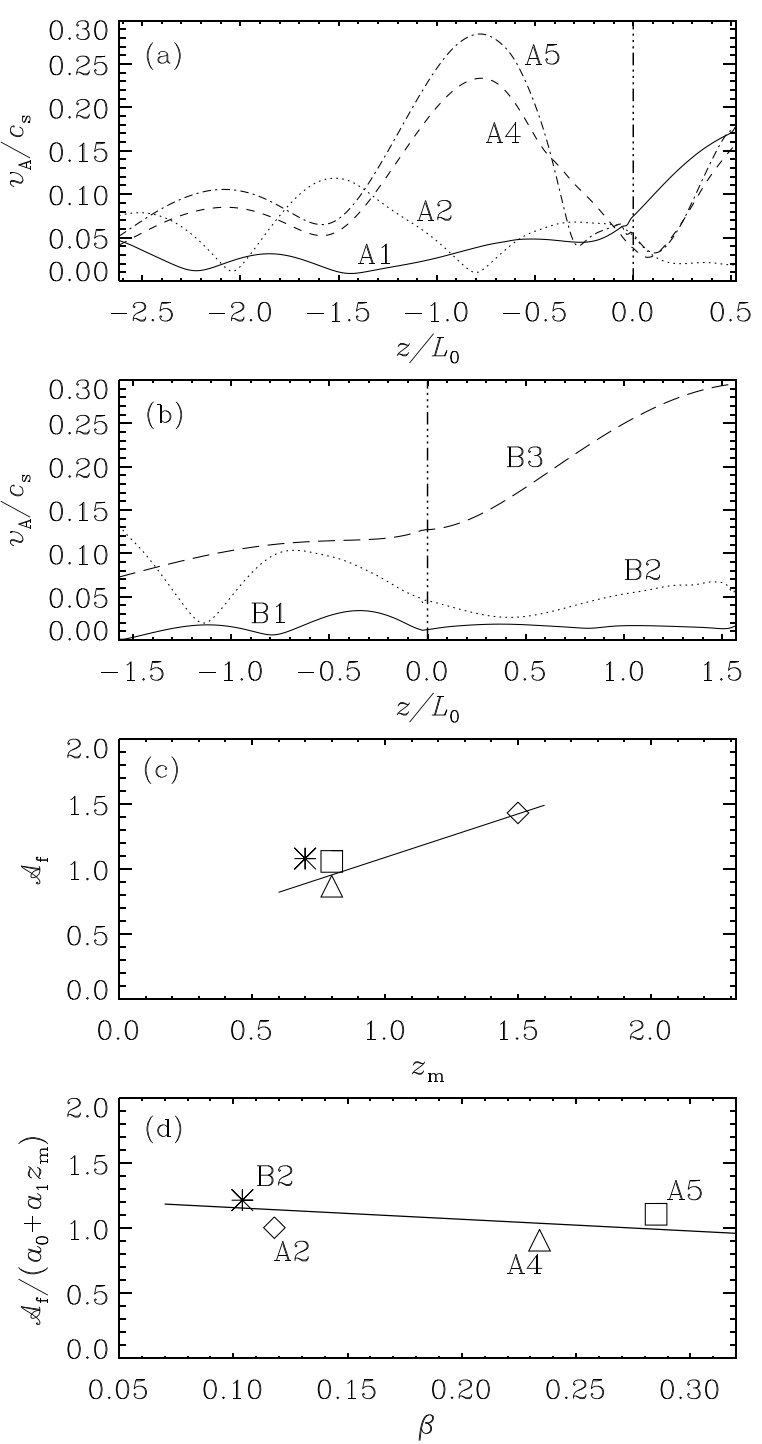

Figure 3. Vertical profiles of the ratio of Alfvén to sound speed, $v_{\mathrm{A}} / c_{\mathrm{S}}$, in the upper two panels; dash-dotted line: position of the interface. $\mathcal{A}_{\mathrm{f}}$ as a function of $z_{\mathrm{m}}$ in panel (c) showing the fit $\mathcal{A}_{\mathrm{f}}=a_{0}+a_{1} z_{\mathrm{m}}$ with $a_{0}=0.42$ and $a_{1}=0.67$ for all values of $\beta$ (solid line); $\mathcal{A}_{\mathrm{f}}$ compensated by this fit as a function of $\beta$ in panel (d).

The described eigenmodes correspond to Bloch waves being bounded solutions of the stationary Schrödinger equation with a periodic potential. According to Bloch's theorem, they must have the form $c_{+} F(x) \exp i k_{0} x+c_{-} F(-x) \exp \left(-i k_{0} x\right)$, where $F(x)$ is a function with the same periodicity as the potential and $k_{0}$ is the so-called Bloch wavenumber (Berton \& Heyvaerts 1987). For our conditions, $k_{0} / k_{1}=l$ and can hence only adopt integers from 0 to $N / 2$.

\section{RESULTS}

To demonstrate the effects of non-uniformity of the magnetic field, we study two types of cases: for the first one, the domain is asymmetric with respect to the interface $\left(L_{z, \mathrm{~d}} / L_{z, \mathrm{u}}=5\right.$; Runs $\mathrm{A} 1-\mathrm{A} 5)$, while for the second, it is symmetric $\left(L_{z, \mathrm{~d}}=L_{z, \mathrm{u}}\right.$; Runs B1-B3).

The corresponding $k \omega$ diagrams are shown in Figure 4. Similar to the non-magnetic or weakly magnetized cases studied by SBCR, we see $p$-modes above the line $\omega=c_{\mathrm{sd}} k_{x}$ with an apparent discontinuity at $\omega=c_{\mathrm{su}} k_{x}$, and indications of $g$-modes at $\widetilde{k}_{x}<4$ and $\widetilde{\omega}=0.5-0.8$. However, the $f$-mode now fans out and spans a trumpet-shaped structure around the nonmagnetic $f$-mode frequency $\omega_{\mathrm{f}}=\sqrt{g k_{x}}$. The more the magnetic field is pushed toward the bottom of the domain, the more asymmetric this expansion appears to be with respect to the $f$-mode in the unmagnetized case.

To quantify the fanning out of the $f$-mode, we denote the upper and lower edges of the fan by $\omega_{\mathrm{f}+}\left(k_{x}\right)$ and $\omega_{\mathrm{f}-}\left(k_{x}\right)$, respectively. In general, the fan is asymmetric with respect to the classical $f$-mode $\left(\omega_{\mathrm{f}}\right)$. Let us represent this asymmetry at any given $k_{x}$ by $\mathcal{A}_{\mathrm{f}}=\Delta \omega_{\mathrm{f}+} / \Delta \omega_{\mathrm{f}-}$, where $\Delta \omega_{\mathrm{f} \pm}=\left|\omega_{\mathrm{f} \pm}-\omega_{\mathrm{f}}\right|$ are the frequency spreads above and below $\omega_{\mathrm{f}}$; see Table 1 for $\Delta \omega_{\mathrm{f} \pm} / \omega_{\mathrm{f}}$ at $\tilde{k}_{x}=9$. We find that at $\widetilde{k}_{x}=15$, the total relative spread, $\left(\Delta \omega_{\mathrm{f}_{+}}+\Delta \omega_{\mathrm{f}-}\right) / \omega_{\mathrm{f}}$, can be as large as 1.1 , which further increases with increasing $\beta$ for fixed $z_{\mathrm{m}}$; see Runs A4 and A5 in Figure 4. Note that $\mathcal{A}_{\mathrm{f}}$ can take values both larger and smaller than unity, as may be seen by comparing Run A2 with Runs A4 or A5 in Figure 4; see also Table 1 and Figure 3. Additional experiments have shown that when we increase the value of $\beta$ further, the power above and below the $\omega_{\mathrm{f}}$ line increases, but the mode profile becomes more diffuse and the edges of the fan less sharp.

In addition, we see as a qualitatively new feature a regular pattern of vertical stripes at multiples of $2 \widetilde{k}_{x}^{B}$ all the way up to $\widetilde{k}_{x}=14$, which appears unconnected with the $f$-mode. (In the spectra of $\boldsymbol{B}$, the stripes appear at odd multiples of $\widetilde{k}_{x}^{B}$.) They are absent if $\boldsymbol{B}_{0}$ is independent of $z\left(k_{z}^{B}=0\right.$; not shown), but most pronounced when the magnetic field is concentrated in the lower part of the domain; see Figure 3(a) for Runs A4 and A5 as well as Figure 3(b) for Run B2. These are also the cases in which the $f$-mode appears most fanned out. The stripes appear weaker when the magnetic field is symmetric about the interface at $z=0$ (Run B3) or when it is generally weak (Runs A1, A2, and B1). Given that they are persistent after switching off the random hydrodynamic forcing (see Run A5f0 in Figure 4), they can be identified to indicate at least one unstable eigensolution. Note that for a fixed $\omega$ an infinitude of $k_{x}$ belongs to the same eigenmode. The discrete $\omega$ spots within each stripe may either belong to different unstable eigenmodes or represent overtones of a single mode. Given that the ratio $|\boldsymbol{\nabla} \cdot \boldsymbol{u}| /|\boldsymbol{\nabla} \times \boldsymbol{u}|$ in Run A5f0 is small $(<0.01)$ everywhere within the domain, we conclude that the velocity field of the stripes is in that sense close to solenoidal. Furthermore, as their occurrence and amplitude are strongly dependent on the strength of $\boldsymbol{B}_{0}$, we propose to consider them as shear Alfvén modes having become unstable due to the inhomogeneity of $\boldsymbol{B}_{0}$. A similar transition is observed in whistler waves which become unstable for suitably non-uniform background fields (Rheinhardt \& Geppert 2002).

Remarkably, the unstable mode(s) excite $f$-modes, but without fanning them out, whereas $p$-modes remain unexcited (Panel A5f0). Comparing panels A5 and A5f0 suggests that the fanning out requires not just a non-uniform magnetic field, but also the presence of random forcing. However, the fact that A5f0 exhibits only a single line and no fan might indicate a physical difference between the fan and the regular $f$-mode. We also note that the expansion of the $f$-mode can still be seen when the vertical stripes are weak or absent (especially in Run A2), but in Run $\mathrm{B} 1$, where the field is less deep and the domain symmetric about $z=0$, the $f$-mode lacks a clear trumpet shape.

Another qualitatively new feature compared to the case of a uniform background field is the occurrence of inclined stripes running from the lower left to the upper right corner of the $k \omega$ diagram, best visible in panel A1 of Figure 4 . They are reminiscent of Alfvén modes. Since they occur only in cases 

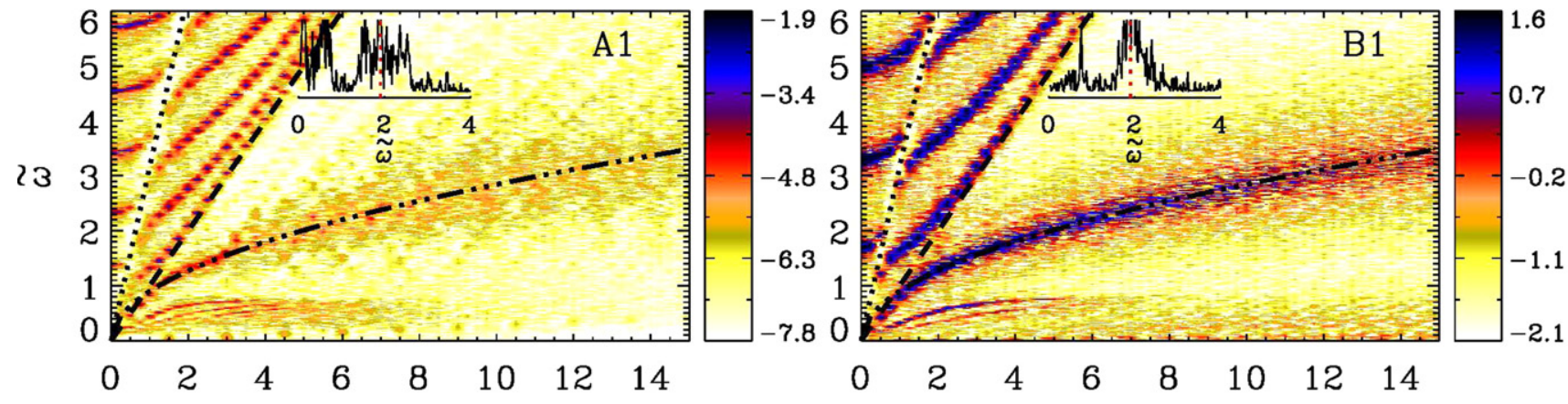

$\begin{array}{llllllll}0 & 2 & 4 & 6 & 8 & 10 & 12 & 14\end{array}$
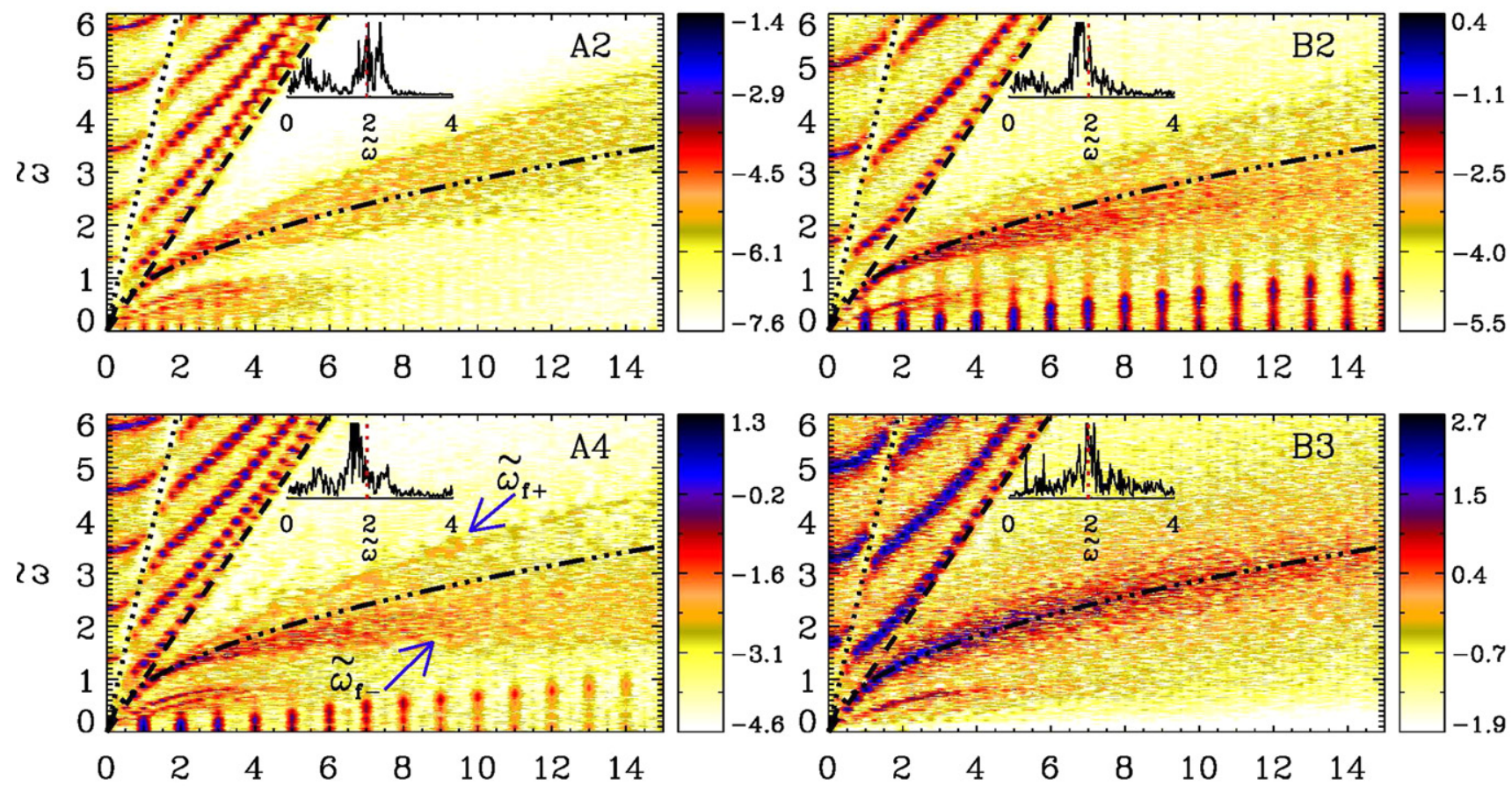

$\begin{array}{llllllll}0 & 2 & 4 & 6 & 8 & 10 & 12 & 14\end{array}$

$\begin{array}{llllllll}0 & 2 & 4 & 6 & 8 & 10 & 12 & 14\end{array}$
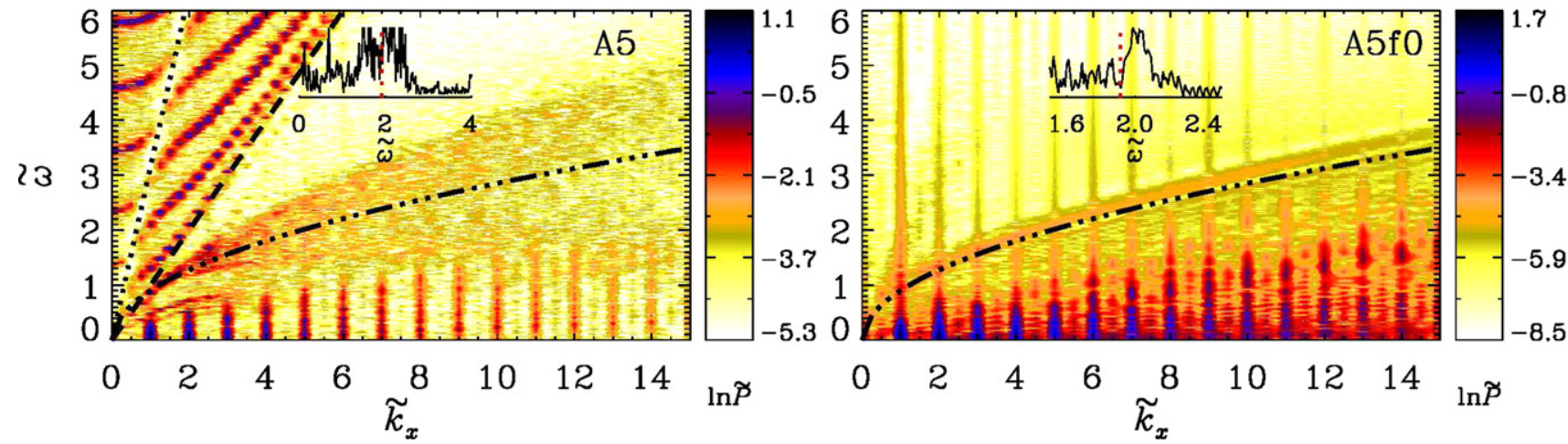

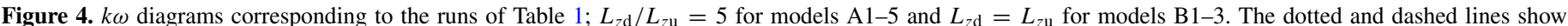

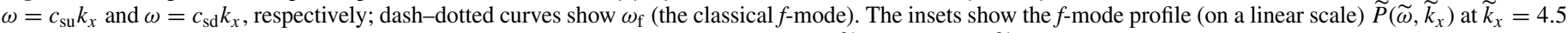
with the red line marking the position of $\omega_{\mathrm{f}}$. In panel $\mathrm{A} 4$, the arrows indicate the upper $\left(\widetilde{\omega}_{\mathrm{f}+}\right)$ and lower $\left(\widetilde{\omega}_{\mathrm{f}-}\right)$ edges of the $f$-mode fan.

(A color version of this figure is available in the online journal.)

where the aforementioned instability is weak or turbulently suppressed, they might be (damped) Alfvén waves modified by the non-uniformity of the background field.

\section{CONCLUSIONS}

The present study was aimed at identifying diagnostic signatures of spatial variability of the magnetic field. Indeed, we find as such characteristic features the fanning out of the $f$-mode and a pattern of vertical stripes in the $k \omega$ diagram which have not been reported in earlier helioseismic studies. Since the use of a non-uniform background field was the only change relative to SBCR, we must conclude that these features are due to this non-uniformity. Our work also suggests that their occurrence is only weakly dependent on Reynolds and Mach numbers.

The fanning out of the $f$-mode is different from the case of capillary waves, where instead a "bifurcation" of the $f$-mode in deep water waves is caused by surface tension (Dias 
$\&$ Kharif 1999). In the present case, the width of the fan and its asymmetry appear to characterize the magnetic field strength. Independent from that, the horizontal variability of the underlying magnetic field is reflected in the presence of vertical stripes in the diagnostic $k \omega$ diagram at even multiples of the horizontal wavenumber of the magnetic field. We have proven that these stripes can be assigned to one or perhaps several unstable eigenmodes, most likely of shear Alfvén type.

The spatial variation of the photospheric field seen in Figure 1 with a wavelength of, say, 100-200 Mm corresponds to a wavenumber of the background field $k_{x}^{B}=0.03-0.06 \mathrm{Mm}^{-1}$, i.e., with $\gamma H_{\mathrm{d}} \approx 0.5 \mathrm{Mm}$, we have $\widetilde{k}_{x}^{B}=0.015-0.03$, which is 10-20 times smaller than the smallest value used in the present work. Given that smaller $\widetilde{k}_{x}^{B}$ yield a sharper fan (see panels A1 and A2 in Figure 4), we might speculate that in solar data the fan could also be sharper, but we have to remember that the assumption of a completely periodic pattern of $\boldsymbol{B}_{0}$ is, of course, too optimistic.

With respect to instrumentation requirements, it is clear that for a possible detection of the fan one would need to reach $\widetilde{k}_{x} \gtrsim 3$ and $1 \lesssim \widetilde{\omega} \lesssim 3$. With $R=700 \mathrm{Mm}$ for the solar radius, this corresponds to $k_{x} \gtrsim 6 \mathrm{Mm}^{-1}$, or $\ell>4200$. This is somewhat above what can currently be achieved. Correspondingly, a cadence of less than a minute would be required. Thus, while it is impossible at present to make a clear case for helioseismic applications, our work has opened an avenue for more targeted searches, both theoretically and observationally.

We thank the referee for useful comments and suggestions. Financial support from the Swedish Research Council under the grants 621-2011-5076 and 2012-5797, the European Research Council under the AstroDyn Research Project 227952, as well as the Research Council of Norway under the FRINATEK grant 231444 are gratefully acknowledged. The computations have been carried out at the National Supercomputer Centres in Linköping and Umeå, as well as the Center for Parallel Computers at the Royal Institute of Technology in Sweden and the Nordic High Performance Computing Center in Iceland.

\section{REFERENCES}

Berton, R., \& Heyvaerts, J. 1987, SoPh, 109, 201

Brandenburg, A. 2005, ApJ, 625, 539

Brandenburg, A., Gressel, O., Jabbari, S., Kleeorin, N., \& Rogachevskii, I. 2014, A\&A, 562, A53

Caligari, P., Moreno-Insertis, F., \& Schüssler, M. 1995, ApJ, 441, 886

Chandrasekhar, S. 1961, Hydrodynamic and Hydromagnetic Stability (New York: Dover)

Daiffallah, K., Abdelatif, T., Bendib, A., Cameron, R., \& Gizon, L. 2011, SoPh, 268, 309

Dias, F., \& Kharif, C. 1999, AnRFM, 31, 301

Felipe, T., Braun, D., Crouch, A., \& Birch, A. 2012, ApJ, 757, 148

Felipe, T., Crouch, A., \& Birch, A. 2013, ApJ, 775, 74

Gizon, L., Birch, A. C., \& Spruit, H. C. 2010, ARA\&A, 48, 289

Hanasoge, S. M., Birch, A. C., Bogdan, T. J., \& Gizon, L. 2008, ApJ, 680, 774

Ilonidis, S., Zhao, J., \& Kosovichev, A. 2011, Sci, 333, 993

Kitchatinov, L. L., \& Mazur, M. V. 2000, SoPh, 191, 325

Kleeorin, N., Mond, M., \& Rogachevskii, I. 1996, A\&A, 307, 293

Miles, A. J., Allen, H. R., \& Roberts, B. 1992, SoPh, 141, 235

Miles, A. J., \& Roberts, B. 1989, SoPh, 119, 257

Miles, A. J., \& Roberts, B. 1992, SoPh, 141, 205

Murawski, K., \& Roberts, B. 1993, A\&A, 272, 595

Parker, E. N. 1975, ApJ, 198, 205

Rae, I. C., \& Roberts, B. 1981, GApFD, 18, 197

Rheinhardt, M., \& Geppert, U. 2002, PhRvL, 88, 101103

Roberts, B. 1981, SoPh, 69, 27

Singh, N. K., Brandenburg, A., Chitre, S. M., \& Rheinhardt, M. 2014, MNRAS, submitted (arXiv:1404.3246) (SBCR)

Tao, L., Weiss, N. O., Brownjohn, D. P., \& Proctor, M. R. E. 1998, ApJL, 496, L39 